\title{
ON THE DEFINITION OF STRESS RATE
}

\author{
By E. F. MASUR (The University of Michigan)
}

The concept of stress rate has been the subject of several current papers. Prager [1] has introduced a discussion of some of the proposed definitions from a Cartesian spatial point of view and, in reviewing their suitability for use in the constitutive equations of plasticity, has arrived at a decided preference for Jaumann's [2] definition. Naghdi and Wainwright [3] have generalized the concept of tensor rate in such a way as to include the definitions mentioned by Prager as special cases. Convected coordinates have been employed by Sedov [4] in a discussion of Prager's results. In continuing the approach adopted by Sedov we investigate in the present note the connection between the various stress rates and the concept of a convected derivative introduced by Oldroyd [5]. Additional arguments in favor of Jaumann's definition are established; moreover, a modification of the latter is proposed in connection with the constitutive equations of plasticity.

In order to make this note reasonably self-contained, a brief resumé of Oldroyd's derivation is given in what follows. Let $x_{i}$ be the instantaneous Cartesian coordinates of a particle ${ }^{2}$ and let $a^{\alpha}$ represent a convected coordinate system defined by $x_{i}=x_{i}\left(a^{\alpha}, t\right)$ in such a way that the material coordinates $a^{\alpha}$ of a particle are time independent. Let $\sigma^{\alpha \beta}$, the contravariant components of stress relative to the material coordinate system in its instantaneous position, be considered functions of the material coordinates $a^{\alpha}$ and of the time $t$. The partial derivative of $\sigma^{\alpha \beta}$ with respect to $t$ is called "the convected derivative" by Oldroyd. The components $D_{t} \sigma^{\alpha \beta}$ so defined form the contravariant components of a tensor, since the functional relationship between the assumed convected coordinate system and some other coordinate system which is also convected is independent of time.

We are therefore justified in defining a tensor $\sigma^{\prime \prime \prime}$ by

$$
\sigma^{\prime \prime \prime \alpha \beta}=D_{\imath} \sigma^{\alpha \beta}
$$

and its components relative to the assumed Cartesian spatial system ${ }^{3}$ by

Since

$$
\sigma_{i j}^{\prime \prime \prime}=x_{i, \alpha} x_{i, \beta} \sigma^{\prime \prime \prime \alpha \beta} \text {. }
$$

$$
\sigma_{i j}=x_{i, \alpha} x_{i, \beta} \sigma^{\alpha \beta}
$$

and

$$
D_{\iota} x_{i}=v_{i},
$$

where $v_{i}$ represents the Cartesian components of the velocity vector, we obtain by differentiation of Eq. (3) Oldroyd's definition

$$
\sigma_{i i}^{\prime \prime \prime}=D_{t} \sigma_{i i}-v_{i, k} \sigma_{i k}-v_{i, k} \sigma_{i k} .
$$

${ }^{1}$ Received November 21, 1960; revised manuscript received December 27, 1960. This note is part of a research program sponsored by the National Science Foundation.

${ }^{2}$ An extension to non-Cartesian spatial systems is straightforward, but contributes nothing essential to the purpose of this discussion.

${ }^{3}$ Greek indices refer to the convected coordinate system and Latin indices to the fixed Cartesian system. 
Similarly it is possible to define a tensor $\sigma^{\prime \prime}$ whose covariant components are given by $D_{t} \sigma_{\alpha \beta}$. Note that the tensor so obtained is in general not the same as the one defined in Eq. (1) (that is, $\sigma_{\alpha \beta}^{\prime \prime}$ and $g_{\alpha \gamma} g_{\beta \lambda} \sigma^{\prime \prime \prime \gamma \lambda}$ are in general different), because the metric tensor $g_{\alpha \beta}$ is time dependent, hence convected time differentiation and lowering and raising of indices are not commutable operations ${ }^{4}$. If the Cartesian components of $\sigma^{\prime \prime}$ are obtained by a process similar to the one employed above, we are led to the definitions of Cotter-Rivlin [8]. Furthermore, if the same process is applied to the Kirchhoff tensor, then the use of the contravariant components leads to Truesdell's [7] definition, while an analogous derivation can be carried out without difficulty in connection with the covariant components.

The four stress rate definitions introduced thus far are rejected by Prager on the basis that their vanishing does not make the stress invariants stationary; consequently their use in the constitutive equations of plasticity leads to complications. This becomes immediately apparent in a convected coordinate system since the use of either contravariant or covariant stress components necessitates the introduction of the time dependent metric tensor in the invariants; the latter are stationary in the presence of stationary stress components only in the case of locally rigid body motion.

This argument for rejection can be strengthened further by the (not unrelated) consideration that the establishment of the constitutive equations of plasticity may make it necessary to separate the stress rate tensor into its isotropic and deviatoric contributions. Moreover, it is reasonable to demand that the vanishing of the stress rates make the deviatoric stress components stationary.

This is not true for any of the definitions introduced thus far. For example, if the contravariant components of the stress deviator, which are defined by

$$
s^{\alpha \beta}=\sigma^{\alpha \beta}-\frac{1}{3} \sigma^{\gamma \lambda} g_{\gamma \lambda} g^{\alpha \beta}
$$

are used for the definition of the stress deviator rate ${ }^{5}$

$$
s^{\prime \prime \prime \alpha \beta}=D_{t} s^{\alpha \beta}
$$

then it follows that $\sigma^{\prime \prime \prime}=0$ does not necessarily imply $s^{\prime \prime \prime}=0$. Conversely, with the introduction of the strain rate tensor $\epsilon$ by means of

$$
2 \epsilon_{\alpha \beta}=v_{\alpha, \beta}+v_{\beta, \alpha}=D_{\imath} g_{\alpha \beta}
$$

the vanishing of $s^{\prime \prime \prime}$ implies

$$
\sigma_{\alpha}^{\prime \prime \prime}=\frac{1}{3} \sigma_{\gamma}^{\prime \prime \prime}{ }_{\gamma}^{\prime \gamma} \delta_{\alpha}^{\beta}-\frac{2}{3} \sigma_{\gamma}^{\gamma}\left(\epsilon_{\alpha}^{\beta}-\frac{1}{3} \epsilon_{\lambda}^{\lambda} \delta_{\alpha}^{\beta}\right) .
$$

It is seen that for this case $\sigma^{\prime \prime \prime}$ is not isotropic, as it should be, unless $\epsilon$ is isotropic too.

The objections raised against the stress rate definitions introduced above become

\footnotetext{
"It is equally valid to refer to $\sigma$ "1 and to $\sigma$ " (or to any suitably normalized combination thereof) as a "convected derivative" of $\sigma$; any rate tensor so defined clearly satisfies the requirement of being insensitive to rigid body motion. The conceptual significance of this ambiguity has apparently not been fully explored by Oldroyd [5], [6] or Truesdell [7], but is implicit in [4].

${ }^{5}$ Note that this defines the rate of the stress deviator, which is not equal to the deviator of the stress rate.
} 
invalid with the use of the mixed components. We therefore define the unsymmetric tensors ${ }^{*} \sigma^{\prime}$ and ${ }^{* *} \sigma^{\prime}$ by means of

$$
\begin{aligned}
*{\sigma_{\alpha}^{\prime \beta}}^{\beta} & =D_{t} \sigma_{\alpha}^{\beta} \\
* *_{\sigma_{\alpha}^{\prime}}^{\beta} & =D_{t}{\sigma^{\beta}}_{\alpha}
\end{aligned}
$$

and the symmetric tensor ${ }^{6}$

$$
\sigma^{\prime}=\frac{1}{2}\left(* \sigma^{\prime}+* * \sigma^{\prime}\right)
$$

The Cartesian components of this tensor are given by

$$
\sigma_{i j}^{\prime}=D_{t} \sigma_{i j}-\omega_{k i} \sigma_{i k}-\omega_{k j} \sigma_{i k}
$$

in which

$$
\omega_{i i}=\frac{1}{2}\left(v_{i, i}-v_{i, i}\right)
$$

are the components of the skew-symmetric vorticity tensor.

It is seen that $\sigma^{\prime}$ is Jaumann's definition; in other words, the artifice of introducing a local rigid coordinate system moving with the particle as employed by Jaumann and again recently by Oldroyd [6] is equivalent to the use of the symmetrized convected time derivative of the mixed components. Note also that $\sigma^{\prime}$ is the mean of $\sigma^{\prime \prime}$ and $\sigma^{\prime \prime \prime}$. An analogous definition applied to vectors leads to a rate definition which is in agreement with elementary concepts.

Under certain circumstances, the use of a weighted tensor in the constitutive equations may be more convenient. For example, Drucker's argument [9] establishing the convexity of the plastic loading function may lead to the conclusion that this function should be expressed in terms of the Kirchhoff tensor components, since the latter govern the rate of work per fixed unit of mass (rather than that per time dependent unit of volume). The equivalent Cartesian stress rate, referred to the instantaneous system, is given by

$$
\sigma^{I V}{ }_{i j}=D_{t} \sigma_{i j}-\omega_{k i} \sigma_{j k}-\omega_{k i} \sigma_{i k}+v_{k, k} \sigma_{i j}
$$

which is acceptable in the light of the preceding discussion.

It may be of some interest to discuss briefly the question of the definition of the strain rate. Offhand, it appears as if similar arguments should make it necessary to define the latter also in terms of the mixed components. This is actually not the case since the definition of the strain tensor $\eta_{\alpha \beta}$ itself employs the metric tensor and, consequently, some of the resulting relationships become somewhat simpler. In fact, with the strain deviator defined by

$$
h_{\alpha \beta}=\eta_{\alpha \beta}-\frac{1}{3} \eta_{\gamma \lambda} g^{\gamma \lambda} g_{\alpha \beta}
$$

then its rate, in view of Eq. (8), takes the form

$$
e_{\alpha \beta}=D_{\iota} h_{\alpha \beta}=\left(\epsilon_{\alpha \beta}-\frac{1}{3} \epsilon_{\gamma}^{\gamma} g_{\alpha \beta}\right)-\frac{2}{3}\left(\eta_{\gamma}^{\gamma} \epsilon_{\alpha \beta}-\eta^{\gamma \lambda} \epsilon_{\gamma \lambda} g_{\alpha \beta}\right) \text {. }
$$

${ }^{6} \mathrm{Of}$ course the symmetry of $\sigma^{\prime}$ is a consequence of that of $\sigma$. Similarly Eq. (11) defines an antisymmetric tensor if the original tensor is also antisymmetric. In particular, if the operation is performed on $\omega$ itself, then (as is plausible from elementary considerations) the material time derivative coincides with the convected derivative. This fact is useful in the determination of higher order rates. 
In this case the vanishing of the strain rate components $\epsilon_{\alpha \beta}$ implies also that the deviator components $h_{\alpha \beta}$ are stationary. Moreover, a limited inverse is also true; that is, the vanishing of $e_{\alpha \beta}$ implies that the strain rate is isotropic.

\title{
REFERENCES
}

1. W. Prager, An elementary diccussion of definitions of strtss rate, Quart. Appl. Math. 18, 403-407 (1961)

2 G. Jaumann, Geschlossenes System physikalischer und chemischer Differenzialgesetze, Sitzungsber. Akad. Wiss. Wien (2a) 120, 385 (1911)

3. P. M. Naghdi and W. L. Wainwright, On the time derivative of tensors in mechanics of continua, Tech. Rept. No. 11, NR 064-436, Univ. of California, Berkeley, Calif. To be published in this journal

4. L. I. Sedov, Different definitions of the rate of change of a tensor, (Poniatiia raznykh skorostei izmeneniia tenzorov) Prikl. Mat. Mekh. 24, 393 (1960)

5. J. G. Oldroyd, On the formulation of the rheological equations of state, Proc. Roy. Soc. (A) 200, 523 (1950)

6. J. G. Oldroyd, Non-Newtonian effects in steady motion of some idealized elastico-viscous liquids, Proc. Roy. Soc. (A) 245, 278 (1958)

7. C. Truesdell, Elasticity and fluid dynamics, J. Ratl. Mech. Analysis 2, 593 (1953)

8. B. A. Cotter and R. S. Rivlin, Tensors associated with time-dependent stress, Quart. Appl. Math. 13, 177 (1955)

9. D. C. Drucker, A more fundamental approach to plastic stress-strain relations, Proc. First U. S. Natl. Congr. Appl. Mech., Am. Soc. Mech. Engrs., p. 487 (1952)

\section{STEADY SPHEROIDAL VORTICES-MORE EXACT SOLUTIONS TO THE NAVIER-STOKES EQUATION*}

\author{
BY VIVIAN O'BRIEN (Applied Physics Laboratory, The Johns Hopkins University)
}

\begin{abstract}
The vorticity equation, the curl of the Navier-Stokes equation, is considered in ellipsoidal coordinates. The steady spheroidal vortex solutions are demonstrated as examples of a class of exact flow solutions characterized by a simple linear vorticity distribution.

Introduction. The Navier-Stokes equation expresses the conservation of momentum in a form suitable for continuum fluid mechanics. For incompressible fluid flow the system of the continuity equation plus the Navier-Stokes equation has a solution that is completely determined, in principle. However, solutions that satisfy the equations exactly are rare due to the non-linear convective term. One such solution that should be included in the meager list of exact solutions is the Hill-Hadamard spherical vortex.

The spherical vortex is an example of a class of steady axi-symmetric flow solutions consisting of a rotational part where the vorticity is proportional to the distance from the axis and an irrotational part. Other examples of this class will be given, in particular, the steady oblate spheroidal vortices which appear to be physically significant.

Hill-Hadamard spherical vortex. Hill [1] showed in 1894 that the stream function

$$
\psi=\sin ^{2} \theta\left(A r^{2}+B r^{4}\right)
$$

${ }^{*}$ Received December 5, 1960. Supported by the Bureau of Naval Weapons, Department of the Navy, under Contract NOrd 7386.
\end{abstract}

